\title{
Degree of Approximationof Functionsby Newly Defined Polynomials onan unbounded interval
}

\author{
Anwar Habib \\ Departmentof General Studies \\ JubailIndustrial College, KSA
}

ABSTRACT : Chlodovsky(1937) has proved the theorem 1.1 \& 1.2 for Bernstein Polynomials $B_{n}(x)=B_{n}^{f}\left(x ; b_{n}\right)=\sum_{k=0}^{n} f\left(\frac{b_{n} k}{n}\right)\left(\begin{array}{l}n \\ k\end{array}\right)\left(\frac{x}{b_{n}}\right)^{k}\left(1-\frac{x}{b_{n}}\right)^{n-k}$ on an unbounded interval.

The object of this paper is to extend the above theorems for newly defined polynomials

on an unbounded interval.

$$
A_{n}(x)=A_{n}^{f}\left(x, \alpha ; b_{n}\right)=(n+1) \sum_{k=0}^{n}\left\{\int_{\frac{k}{n+1}}^{\frac{k+1}{n+1}} f(t b) d t\right\} p_{n, k}\left(\frac{x}{b_{n}} ; \alpha\right)
$$

Keywords:Bernstein Polynomials, Lebsgue Integrable function, $L_{1}$ norm, generating function, Modified Polynomials

\section{Introduction \& results}

If $\mathrm{f}(\mathrm{x})$ is a function defined on $[0,1]$, the Bernstein polynomial $\mathrm{B}_{\mathrm{n}}{ }^{\mathrm{f}}(\mathrm{x})$ of $\mathrm{f}$ is $\mathrm{B}_{\mathrm{n}}{ }^{\mathrm{f}}(\mathrm{x})=\sum_{k=0}^{n} f\left(\frac{k}{n}\right) p_{n, k}(x)$, where $p_{n, k}(x)=\left(\begin{array}{l}n \\ k\end{array}\right)(x)^{k}(1-x)^{n-k}$.

If the function $\mathrm{f}(\mathrm{x})$ defined in the interval $(0, \mathrm{~b}), \mathrm{b}>0$. The Bernstein polynomialB $\mathrm{B}_{\mathrm{f}} \mathrm{f}(\mathrm{x} ; \mathrm{b})$ for this interval is given by

$\mathrm{B}_{\mathrm{n}}(\mathrm{x})=\mathrm{B}_{\mathrm{n}}{ }^{\mathrm{f}}(\mathrm{x} ; \mathrm{b})=\sum_{k=0}^{n} f\left(\frac{\mathrm{b}}{n}\right)\left(\begin{array}{l}n \\ k\end{array}\right)\left(\frac{x}{\mathrm{~b}}\right)^{k}\left(1-\frac{x}{\mathrm{~b}}\right)^{n-k}$

Further a small modification of the Bernstein polynomial due toKantorovich [2] and Anwar\&Umar [3] makes it possible to approximate Lebesgue integrable function in $\mathrm{L}_{1}$ norm by a newly defined polynomial

$A_{n}^{\alpha}(\mathrm{f}, \mathrm{x})=(n+1) \sum_{k=0}^{n}\left\{\int_{\frac{k}{n+1}}^{\frac{k+1}{n+1}} f(t) d t\right\} p_{n, k}(x ; \alpha)$

where

$$
p_{n, k}(\mathrm{x} ; \alpha)=\left(\begin{array}{l}
\mathrm{n} \\
\mathrm{k}
\end{array}\right) \frac{\mathrm{x}(\mathrm{x}+\mathrm{k} \alpha)^{\mathrm{k}-1}(1-\mathrm{x}+(\mathrm{n}-\mathrm{k}) \alpha)^{\mathrm{n}-\mathrm{k}}}{(1+\mathrm{n} \alpha)^{\mathrm{n}}}
$$

such that $\sum p_{n, k}(\mathrm{x} ; \alpha)=1$

Let the function $\mathrm{f}(\mathrm{x})$ be defined on the interval $(0, \mathrm{~b}), \mathrm{b}>0$. To obtain a modified polynomial $A_{n}^{f}(\mathrm{x}, \alpha ; b)$ for this interval, we make the substitution

$\mathrm{y}=\mathrm{xb}^{-1}$ in the polynomial $A_{n}^{\Phi}(\mathrm{y})$ of the function $\Phi(y)=f(b y), 0 \leq \mathrm{y} \leq 1$

and obtain in this way

$A_{n}(\mathrm{x})=A_{n}^{f}(\mathrm{x}, \alpha ; \mathrm{b})=(n+1) \sum_{\mathrm{d}_{k=0}}^{n}\left\{\int_{\frac{k}{n+1}}^{\frac{k+1}{n+1}} f(t b) d t\right\} p_{n, k}\left(\frac{x}{b} ; \alpha\right)$---- (1.4)

where

$p_{n, k}\left(\frac{\mathrm{x}}{\mathrm{b}} ; \alpha\right)=\left(\begin{array}{l}\mathrm{n} \\ \mathrm{k}\end{array}\right) \frac{\left(\frac{\mathrm{x}}{\mathrm{b}}\right)\left(\frac{\mathrm{x}}{\mathrm{b}}+\mathrm{k} \alpha\right)^{\mathrm{k}}\left(1-\frac{\mathrm{x}}{\mathrm{b}}+(\mathrm{n}-\mathrm{k}) \alpha\right)^{\mathrm{n}-\mathrm{k}}}{(1+\mathrm{n} \alpha)^{\mathrm{n}}}$

Chlodovsky (1937) has proved the theorem by assuming $\mathrm{b}=b_{n}$ is a function of $\mathrm{n}$, which increases to $+\infty$ with $\mathrm{n}$ and $\mathrm{f}(\mathrm{x})$ defined in the infinite interval $0 \leq \mathrm{x}<\infty$.

Theorem 1.1:- If $b_{n}=0(\mathrm{n})$ and the function $\mathrm{f}(\mathrm{x})$ is bounded in $[0,+\infty)$, say $|f(x)| \leq \mathrm{M}$, then then $\mathrm{B}_{\mathrm{n}}(\mathrm{x}) \rightarrow \mathrm{f}(\mathrm{x})$ holdsat any point of continuity of the function $\mathrm{f}(\mathrm{x})$. 
Theorem 1.2:- If $b_{n}=0(\mathrm{n})$

and

$$
\mathrm{M}\left(b_{n}\right) e^{-\mathrm{an} / \mathrm{bn}} \rightarrow 0,
$$

for each $\alpha>0$, then $B_{n}(x) \rightarrow f(x)$ holds at each point of continuity of the function $f(x)$.

In this paper our object is to improve the above results by taking the new polynomial $A_{n}(\mathrm{x})$ instead of $\mathrm{B}_{\mathrm{n}}(\mathrm{x})$ which may be stated as follows

Theorem 1.3:- If $b_{n}=0(\mathrm{n})$ and the function $\mathrm{f}(\mathrm{x})$ is bounded lebesgue integrable in $[0,+\infty)$, say $|f(x)| \leq \mathrm{M}$, then $A_{n}(x) \rightarrow f(x)$ holdsat any point of continuity of the function $f(x)$.

Theorem 1.4:- If $b_{n}=0(\mathrm{n})$

and

$$
\mathrm{M}\left(b_{n}\right) e^{-\beta \mathrm{n} / b_{n}} \rightarrow 0,
$$

for each $\beta>0$, then $A_{n}(x) \rightarrow f(x)$ holds at each point of continuity of the function $f(x)$.

\section{Lemmas}

In order to proofour result we need the following Lemmas

Lemma2.1:[3] For all values of $x \in[0,1]$ and for $\alpha=\alpha_{n}=0\left(\frac{1}{n}\right)$

We have $(n+1) \sum_{k=0}^{n}\left\{\int_{\frac{k}{n+1}}^{\frac{k+1}{n+1}}(t-x)^{2} d t\right\} p_{n, k}(x ; \alpha) \leq \frac{x(1-x)}{n}$.

Lemma 2.2: If $0 \leq x \leq 1$, the inequality,

$$
0 \leq \mathrm{Z} \leq \frac{3}{2}\left(\frac{x(1-x)}{n}\right)^{\frac{1}{2}}
$$

Implies

$(n+1) \sum_{|t-x| \geq 2 z\left(\frac{x(1-x)}{n}\right)^{\frac{1}{2}}} \int_{\frac{k}{n+1}}^{\frac{k+1}{n+1}} p_{n, k}(x) d t \leq 2 e^{-z^{2}}$

Proof of lemma 2.2: $\quad$ Let $\Phi$ be the generating function of the polynomial

$$
\mathrm{T}=\sum_{k=0}^{n}(k-n x) p_{n, k}(x: \alpha),
$$

which may be defined as

$$
\begin{aligned}
\Phi= & \Phi_{n}(u, s)=\sum_{s=0}^{\infty} \frac{1}{s !} T_{n s}(x) u^{s} \\
= & \sum_{k=0}^{n} p_{n, k}(x ; \alpha) \sum_{s=0}^{\infty} \frac{1}{s !}(k-n x)^{s} u^{s} \\
= & \sum_{k=0}^{n} e^{u(k-n x)}\left(\frac{\mathrm{n}}{\mathrm{k}}\right) \frac{\mathrm{x}(\mathrm{x}+\mathrm{k} \alpha)^{\mathrm{k}-1}(1-\mathrm{x}+(\mathrm{n}-\mathrm{k}) \alpha)^{\mathrm{n}-\mathrm{k}}}{(1+\mathrm{n} \alpha)^{\mathrm{n}}} \\
= & \frac{e^{-n x u}}{(1+\mathrm{n} \alpha)^{\mathrm{n}} \mathrm{n}}(1-\mathrm{x}+\mathrm{n} \alpha)^{\mathrm{n}}+n x(1-\mathrm{x}+(\mathrm{n}-1) \alpha)^{\mathrm{n}-1} e^{u} \\
& \quad+\frac{n(n-1)}{2 !} x(\mathrm{x}+2 \alpha)(1-\mathrm{x}+(\mathrm{n}-2) \alpha)^{\mathrm{n}-2} e^{2 u}+\cdots \cdots \cdots+x(\mathrm{x}+\mathrm{n} \alpha)^{n-1} e^{n u} \\
\Phi= & e^{-n x u}\left(1-\mathrm{x}+\mathrm{x} e^{u}\right)^{\mathrm{n}}, \text { for } \alpha=\alpha_{n}=0\left(\frac{1}{\mathrm{n}}\right)
\end{aligned}
$$

and therefore

$$
\Phi=\left[e^{-x u}\left(1-\mathrm{x}+\mathrm{x} e^{u}\right)\right]^{\mathrm{n}}
$$

To prove our result we first show that for $|u| \leq \frac{3}{2}$, the inequality

holds.

$$
\Phi \leq \exp \left\{\sin x(1-x) u^{2}\right\}
$$

For (2.3) can be written as

$$
\left.\Phi=\left[x e^{u(1-x)}+(1-\mathrm{x}) e^{-u x}\right)\right]^{\mathrm{n}}
$$


But since

$$
\begin{aligned}
& x e^{u(1-x)}+(1-x) e^{-u x}=\sum_{v=0}^{\infty} \frac{u^{v}}{v !}\left[x(1-x)+(1-x)(-x)^{v}\right] \\
& \leq 1+\sum_{v=2}^{\infty} \frac{u^{v}}{v !}\left[x(1-x)+(1-x)(-x)^{v}\right] \\
& \leq 1+x(1-x) \sum_{v=2}^{\infty} \frac{|u|^{v}}{v !} \\
& \leq 1+x(1-x) \frac{u^{2}}{2}\left(1+\frac{|u|}{3}+\frac{\left|u^{2}\right|}{3^{2}}+\ldots \ldots \ldots \ldots\right) \\
& =1+x(1-x) \frac{u^{2}}{2}\left(1-\frac{1}{3}|u|\right)^{-1} \\
& \leq 1+x(1-x) u^{2} \quad \text { for } \quad|u| \leq \frac{3}{2} \\
& \leq e^{x(1-x) u^{2}} \text { as } e^{k}>k+1
\end{aligned}
$$

and hence

$$
\begin{aligned}
\Phi & \leq\left[e^{x(1-x) u^{2}}\right]^{\mathrm{n}} \\
& =\exp \left\{n x(1-x) u^{2}\right\} \quad \text { which is (2.4). }
\end{aligned}
$$

Therefore if

$$
\psi=\psi_{n}(u, x)=\sum_{k=0}^{n} e^{u|k-n x|} p_{n, k}(x ; \alpha)
$$

then we obtain for $0 \leq \mathrm{u} \leq \frac{3}{2}$

$$
\begin{aligned}
& \psi \leq \psi_{n}(u, x)+\Psi_{n}(-u, x) \text { and therefore, for } \alpha=\alpha_{n}=0\left(\frac{1}{n}\right) \text {, we have } \\
& \psi \leq \exp 2\left\{n x(1-x) u^{2}\right\} \text {. ----- }
\end{aligned}
$$

now we get our required result, we note that for $c \geq 0$ and $u \geq 0$

$$
\begin{gathered}
\left.(n+1) \sum_{c \psi}^{n}(n+1) \sum_{\frac{k}{n+1}}^{\frac{k+1}{n+1}} d t\right) p_{n, k}(x ; \alpha) \\
\left\{\int_{\frac{k}{n+1}}^{\frac{k+1}{n+1}} f(t) d t\right\} e^{u|k-n x|} p_{n, k}(x ; \alpha) \leq \frac{1}{c}
\end{gathered}
$$

Now if we put $\mathrm{c}=\frac{1}{2} z^{2}$, we obtain

$$
(n+1) \sum_{\exp [\llbracket u|k-n x|] \geq \frac{1}{2} e^{z^{2}} \psi}\left(\int_{\frac{k}{n+1}}^{\frac{k+1}{n+1}} d t\right) p_{n, k}(x ; \alpha) \leq 2 e^{-z^{2}}
$$

or

$$
(n+1) \sum_{|k-n x| \geq z^{2} u^{-1}+n x(1-x) u}\left(\int_{\frac{k}{n+1}}^{\frac{k+1}{n+1}} d t\right) p_{n, k}(x ; \alpha) \leq 2 e^{-z^{2}}
$$

since for the given range of $t|k-n x| \sim|t-x|$, we have

$$
(n+1) \sum_{|t-x| \geq z^{2} u^{-1} n^{-1}+n x(1-x) u}\left(\int_{\frac{k}{n+1}}^{\frac{k+1}{n+1}} d t\right) p_{n, k}(x ; \alpha) \leq 2 e^{-z^{2}}
$$

Since 2.1 can be written as

$0 \leq \mathrm{z}[n x(1-x)]^{-\frac{1}{2}} \leq \frac{3}{2}$

But (2.7)holds for $0 \leq \mathrm{u} \leq \frac{3}{2}$ and therefore for $\mathrm{u}=\mathrm{z}[n x(1-x)]^{-\frac{1}{2}}$, we have

$(n+1) \sum_{|t-x| \geq z\left[\frac{x(1-x)}{n}\right]^{\frac{1}{2}}+z\left[\frac{x(1-x)}{n}\right]^{\frac{1}{2}}}\left(\int_{\frac{k}{n+1}}^{\frac{k+1}{n+1}} d t\right) p_{n, k}(x ; \alpha) \leq 2 e^{-z^{2}}$ 
$(n+1) \sum_{|t-x| \geq 2 z\left[\frac{x(1-x)}{n}\right]^{\frac{1}{2}}}\left(\int_{\frac{k}{n+1}}^{\frac{k+1}{n+1}} d t\right) p_{n, k}(x ; \alpha) \leq 2 e^{-z^{2}}$

this completes the proof of lemma.

\section{Proof of theorem 1.3:}

\section{Proof of theorems}

We have

$\left|A_{n}(\mathrm{x})-f(x)\right| \leq(n+1) \sum_{k=0}^{n}\left\{\int_{\frac{k}{n+1}}^{\frac{k+1}{n+1}}|f(t b)-f(x)| d t\right\} p_{n, k}\left(\frac{x}{b} ; \alpha\right)$

Let $\epsilon>0$ be arbitrary and choose infinitesimally small $\delta>0$ such that $\left|f(\mathrm{x})-f\left(x^{\prime}\right)\right|<\epsilon$ for $\left|x-x^{\prime}\right|<\delta$

then

$$
\begin{aligned}
& \left|A_{n}(\mathrm{x})-f(x)\right| \leq(n+1) \sum_{|b n t-x|<\delta}\left\{\int_{\frac{k}{n+1}}^{\frac{k+1}{n+1}}|f(t b)-f(x)| d t\right\} p_{n, k}\left(\frac{x}{b} ; \alpha\right) \\
& +(n+1) \sum_{|b n t-x| \geq \delta}\left\{\int_{\frac{k}{n+1}}^{\frac{k+1}{n+1}}|f(t b)-f(x)| d t\right\} p_{n, k}\left(\frac{x}{b} ; \alpha\right) \\
& =\mathrm{I}_{1}+\mathrm{I}_{2} \\
& \mathrm{I}_{1}=(n+1) \sum_{|b n t-x|<\delta}\left\{\int_{\frac{k}{n+1}}^{\frac{k+1}{n+1}}|f(t b)-f(x)| d t\right\} p_{n, k}\left(\frac{x}{b} ; \alpha\right) \\
& <\epsilon(n+1) \sum_{|b n t-x|<\delta}\left\{\int_{\frac{k}{n+1}}^{\frac{k+1}{n+1}} d t\right\} p_{n, k}\left(\frac{x}{b} ; \alpha\right)
\end{aligned}
$$

To calculate $\mathrm{I}_{2}$, we put $\mathrm{u}=\frac{x}{b_{n}}$ and then we have

$$
\begin{aligned}
& \mathrm{I}_{2}=(n+1) \sum_{|b n t-x| \geq \delta}\left\{\int_{\frac{k}{n+1}}^{\frac{k+1}{n+1}}|f(t b)-f(x)| d t\right\} p_{n, k}\left(\frac{x}{b} ; \alpha\right) \\
& \leq 2 \mathrm{M}(n+1) \sum_{|t-u| \geq \frac{\delta}{b n}}\left\{\int_{\frac{k}{n+1}}^{\frac{k+1}{n+1}} d t\right\} p_{n, k}(u ; \alpha) \\
& \left.\leq 2 M\left(\frac{\delta}{b n}\right)^{-2}(n+1) \sum_{k=0}^{\frac{k+1}{n+1}}(t-u)^{2} d t\right\} p_{n, k}(u ; \alpha)
\end{aligned}
$$

$\leq 2 M\left(\frac{\delta}{b n}\right)^{-2} \frac{u(1-u)}{n}$ for all $\alpha=0\left(\frac{1}{n}\right) b y \operatorname{Lemma}(2.1)$,

$\leq 2 \mathrm{M} \frac{\frac{x}{b n}}{n\left(\frac{\delta}{b n}\right)^{2}}$ for all large $\mathrm{n} \& \alpha=0\left(\frac{1}{\mathrm{n}}\right)$ since $b_{n}=\mathrm{o}(n)$,

$<\epsilon$

\section{Hence}

$\left|A_{n}(\mathrm{x})-f(x)\right| \leq \epsilon+\epsilon=2 \epsilon$

this completes the proof of theorem 1.3.

Proof of theorem 1.4: Proceeding as in theorem 1.3 we obtain 


$$
\left|A_{n}(\mathrm{x})-f(x)\right| \leq \epsilon+2 \mathrm{M}\left(b_{n}\right)(n+1) \sum_{|t-u| \geq \frac{\delta}{b_{n}}}\left\{\int_{\frac{k}{n+1}}^{\frac{k+1}{n+1}} d t\right\} p_{n, k}(u ; \alpha)
$$

The second term can be easily estimated by means of lemma(2.2), if

$\mathrm{z}=\delta_{n}\left(2 b_{n}\right)^{-1}\left(\frac{u(1-u)}{n}\right)^{-\frac{1}{2}}$,

the condition (2.1) satisfied if we assume, for instance, $\delta<2 \mathrm{x}$ and that $\mathrm{n}$ is sufficiently large .

Hence by (1.6) we obtain

$\left|A_{n}(\mathrm{x})-f(x)\right| \leq \epsilon+2 \mathrm{M}\left(b_{n}\right) \exp \left(-\mathrm{z}^{2}\right)$

$$
\begin{aligned}
& =\epsilon+2 \mathrm{M}\left(b_{n}\right) \exp \left\{-\delta^{2} \cdot \mathrm{n}\left[4 \frac{x}{b n}\left(1-\frac{x}{b n}\right)\right]^{-1}\right\} \\
& =\epsilon+\epsilon=2 \epsilon \text { for large } \mathrm{n}
\end{aligned}
$$

this completes the proof of the theorem 1.4.

\section{Conclusion}

In this paper we have improved the results of Chlodovsky by taking the new Modified Polynomials $A_{n}(\mathrm{x})$ instead of Bernstein Polynomials $B_{n}(x)$.

\section{References}

[1] Chlodovsky, I (1937). Sur le development des fonctions definies dans un interval infini en series de polynomes de M S Bernstein composition math, 4,380-93

[2] Kantorovic, L A (1930). Sur certains developpements suivaint les polynomes de la forme de S Bernstein I , II, C R Acad. Sci. USSR , 20,563-68,595- 600

[3] Anwar Habib and S Umar (1980) “On Generalized Bernstein Polynomials” Indian J. pure appl. Math. , 11(2), $177-189$. 